Abstract

\title{
Fast Analysis of Glucose Content in Food Samples Using a Chitosan-GOx/TiO ${ }_{2} \mathrm{NTAs} / \mathrm{Ti}$ Amperometric Biosensor ${ }^{\dagger}$
}

\author{
Margalida Artigues-Cladera, Jordi Abella and Sergi Colominas * \\ Electrochemical Methods Laboratory, Department of Analytical and Applied Chemistry, ETS Institut Químic \\ de Sarrià, Universitat Ramon Llull, 08017 Barcelona, Spain; margalida.artigues@iqs.edu (M.A.-C.); \\ jordi.abella@iqs.edu (J.A.) \\ * Correspondence: sergi.colominas@iqs.es \\ + Presented at the 5th International Symposium on Sensor Science (I3S 2017), Barcelona, Spain, \\ 27-29 September 2017.
}

Published: 20 November 2017

Among all the analytes that are monitored for quality control in the food sector, glucose is assuming a priority role because it has been associated with chronic health consequences. Numerous methods have been reported for glucose analysis in food, however, most of the adopted methods are time consuming or expensive. The development of fast, cheap, practical and selective methods for detecting glucose in food is still a popular research area. In this context, enzymatic-based biosensors can be used to produce easy-to-use, compact and inexpensive analytical tools.

In the present work, an amperometric glucose biosensor based on glucose oxidase (GOx) immobilization onto highly ordered titanium dioxide nanotube arrays $\left(\mathrm{TiO}_{2} \mathrm{NTAs}\right.$ ) was used to determine the glucose content of commercial food samples (soft drinks and soy sauces). The fundamental analytical parameters of the proposed biosensor were evaluated in order to guarantee that the analytical methodology is accurate, specific, reproducible and robust. The obtained results proved sufficient repeatability ( $\mathrm{RSD}=1.9 \%$ ), reproducibility ( $\mathrm{RSD}=2.5 \%$ ), accuracy (recovery between $95 \%$ and $105 \%$ ) and robustness (RSD $=3.3 \%$ ). Finally, the glucose content of different samples was measured using the biosensor and compared with the HPLC value. In the worst case scenario, a deviation smaller than $7 \%$ was obtained from the 11 samples evaluated.

(C) 2017 by the authors. Licensee MDPI, Basel, Switzerland. This article is an open access article distributed under the terms and conditions of the Creative Commons Attribution (CC BY) license (http://creativecommons.org/licenses/by/4.0/). 